\title{
Mild anxiety and depression related to elevated dopamine level in young adults
}

\author{
Ika Fidianingsih*, Titis Nurmasitoh**, Adika Zhulhi Arjana***, \\ Ninda Devita***, and Umatul Khoiriyah ${ }^{* a}$
}

\begin{abstract}
\section{BACKGROUND}

Dopamine is an important neurotransmitter that plays a role in the pathogenesis of anxiety and depression. Dopamine secretion occurs when there is a pleasurable stimulus. Blood dopamine levels have the potential to be developed as biomarkers of depression or anxiety, but previous studies related to the relationship between dopamine levels and levels of anxiety and depression are still controversial. The purpose of this study was to determine the correlation between anxiety and depression score with dopamine level in young adults.
\end{abstract}

\section{METHODS}

This was an observational cross sectional study. A total of 43 subjects aged 18 to 40 years were recruited by consecutive non-random sampling according to inclusion and exclusion criteria. Anxiety and depression were assessed using the Beck anxiety inventory and Beck depression inventoryII questionnaire. Dopamine plasma level was measured using the enzymelinked immunosorbent assay (ELISA). The Spearman rho correlation test was used to analyze the data and $p<0.05$ was considered significant.

\section{RESULTS}

The median dopamine levels in respondents with mild, moderate and severe anxiety were respectively: $77.79 ; 63.43 ; 62.51 \mathrm{ng} / \mathrm{mL}(\mathrm{p}=0.043)$. The median dopamine levels in respondents with reasonable depression, mood disorder, moderate and severe depression were respectively: 79.2; 61,32; 62,51; 60.24 $(\mathrm{p}=0.001)$. Correlation test results showed a weak correlation between dopamine and anxiety $(\mathrm{r}=-0.310)$ and a moderate correlation for depression $(\mathrm{r}=-0.505)$.

\section{CONCLUSION}

Mild anxiety and depression were associated with elevated dopamine level in young adults. Further studies are required to confirm the role of dopamine metabolism in anxiety and depression in young adults.

Keywords : Anxiety, depression, dopamine, ELISA, young adults
*Department of Histology, Faculty of Medicine, Universitas Islam Indonesia, Yogyakarta

**Department of Physiology, Faculty of Medicine, Universitas Islam Indonesia, Yogyakarta ***Faculty of Medicine, Universitas Islam Indonesia, Yogyakarta Department of Medical Education, Faculty of Medicine, Universitas Islam Indonesia, Yogyakarta

\section{Correspondence:}

${ }^{\circledR}$ Umatul Khoiriyah,MD,M.Med.Ed,PhD Faculty of Medicine, Universitas Islam Indonesia Jl. Kaliurang Km14,5

Sleman Yogyakarta, Indonesia Phone: (62)81328801247

Fax: (62)274898444 Ext.2007. Email: umakhoiriyah@uii.ac.id ORCID ID:

https://orcid.org/0000-0001-8645-8482

Date of first submission, November 17, 2018

Date of final revised submission, March 29, 2019

Date of acceptance, March 29, 2019

This open access article is distributed under a Creative Commons AttributionNon Commercial-Share Alike 4.0 International License

Cite this article as: Fidianingsih I, Nurmasitoh T, Arjana AZ, et al. Mild anxiety and depression related to elevated dopamine level in young adults. Univ Med 2019;38:48-55. doi: 10.18051/UnivMed.2019.v38.48-55 


\section{INTRODUCTION}

In line with technological advances, the public becomes more individualistic, and the level of depression and anxiety increases. Worldwide, the number of persons experiencing anxiety and depression from 2005 to 2015 increased by $15 \%$ and $18 \%$, respectively. ${ }^{(1)}$ In Indonesia, the number of mental cases is also on the increase, impacting on an increased national burden and decreased long-term human productivity. Data from Riskesdas 2013 showed that the prevalence of mental-emotional disorders as shown by the symptoms of depression and anxiety for the age group of 15 years and above attained around 14 million persons or $6 \%$ of the total Indonesian population, ${ }^{(2)}$ whereas globally there was in 2015 an estimated 264 million inhabitants (3.6\% of the population) experiencing anxiety and 322 million (4.4\%) experiencing depression. Depression results in a $40 \%$ higher early mortality than in the general population. Worldwide, a total of 788,000 persons in 2015 committed suicide due to depression. ${ }^{(1)}$

There are various manifestations of anxiety disorder, depending on the type of anxiety. According to the Diagnostic and Statistical Manual of Mental Disorders (DSM-5), anxiety disorder may be in the form of mutism, phobia, social anxiety disorder, panic disorder, generalized anxiety disorder, and anxiety from certain illnesses and medications. The complaint of anxiety may be accompanied by somatic symptoms such as palpitations, shortness of breath, pain in the thorax, nausea, perspiration, tremors, choking, and anesthesia. ${ }^{(3)}$ The symptom of anxiety frequently also occurs in patients with depression, although not invariably so. Depression is a mood disorder that causes sadness and affects feeling, thinking, and activities of daily living such as sleeping, eating, or working. ${ }^{(4)} \mathrm{Up}$ to the present, the diagnosis of anxiety and depression is based on subjective symptoms only and there are yet no specific biomarkers to be found, so that there are still patients with undiagnosed depression. ${ }^{(5)}$ Any uncertainty of diagnosis results in the patients not receiving good management and treatment, so that their condition becomes chronic and they are at risk of more severe mental disorders. The biomarkers that are suitable for neuropsychiatric disorders may frequently be obtained from brain tissue or cerebrospinal fluid, but the procedure is relatively more invasive than drawing a blood sample. Therefore, there is a need for the development of blood biomarkers of depression or anxiety. ${ }^{(6)}$ Biomarkers in the form of microRNAs (miRNAs) from blood samples of patients with schizophrenia have been identified to be significantly associated with the diagnosis and clinical manifestations of this disorder.(7) Administration of antidepressants also showed a significant increase in the expression of 28 types of miRNAs and a significant decrease in 2 types of miRNA. ${ }^{(8)}$

The causative factors of anxiety and depression are nearly identical, comprising biologic (e.g. genetics, age, gender), psychological (e.g. cognitive factors, problem solving ability, and convictions), and social factors such as trauma, social support, and social skills. Depression may occur concomitantly with other serious disorders, such as cancer, heart disease, and Parkinson's disease. ${ }^{(4)}$ The precipitating factor of anxiety may be other diseases such as diabetes mellitus. The pathophysiology of anxiety and depression is also much associated with neurotransmitter imbalance in the brain. ${ }^{(3)}$

One of the essential neurotransmitters in the behavioral response to environmental stimulants that plays an important role in the genesis of anxiety and depression is dopamine. Dopamine is a precursor of norepinephrine (noradrenaline), and is the neurotransmitter involved in the regulation of movement, feeling, and motivation, so that an abnormality of the neurotransmitter will be associated with anxiety and depression. dopamine is produced upon stimulation of pleasure from Ltyrosine, that is converted to L-3,4dihydroxyphenylalanine (L-DOPA) to form dopamine by means of the enzyme DOPA decarboxylase, also known as aromatic L-amino acid decarboxylase, which is secreted and binds 
to the dopamine receptors DR1, DR2, DR3, DR4, and DR5. Dopamine can be degraded by means of monoamine oxidase (MAO) and catechol-Omethyltransferase (COMT) into 3,4dihydroxyphenylacetic acid (DOPAC) and 3methoxytyramine (3MT).${ }^{(9)}$ Deficient production of dopamine results in decreased responsiveness to pleasure stimulation.

The neurotransmitter known up to now, i.e. serotonin, has been recommended as the target for treatment of depression by selective serotonin reuptake inhibitors (SSRI), but large-scale clinical trials failed to show improvement. ${ }^{(10)}$ Therefore, the involvement of dopamine in anxiety and depression constitutes an important and novel perspective that should be studied further. The results of existing studies on dopamine and anxiety are still variable, such as that lack of dopamine may induce anxiety, ${ }^{(11)}$ whereas administration of L-DOPA, which is the precursor of dopamine, may improve anxiety. ${ }^{(4)}$ Medications that may lower dopamine function, such as dopamine antagonists, have been reported in connection with the occurrence of symptoms of social anxiety disorder. $^{(12)}$

There are many studies on the involvement of dopamine in depression and anxiety that are still conducted in experimental animals, since they involve the dopaminergic system in the brain. Depression is frequently associated with decreased dopamine secretion in the synaptic cleft of the accumbent nucleus or the ventral tegmental area, leading to decreased response to pleasure stimuli. ${ }^{(13)}$ Although other studies showed the opposite, i.e. rats with depression and psychiatric disorders, experience an increase in dopamine level in certain regions of the brain, in comparison with normal rats. ${ }^{(14)}$

The relationship between blood dopamine level and depression in humans is still subject to controversy. Studies on patients with depression showed that blood dopamine levels are lower than in persons without depression. ${ }^{(15)}$ However, other studies showed that on the contrary dopamine levels are higher in persons experiencing depression. ${ }^{(16)}$ Still other studies showed that dopamine levels are similar in patients with and without depression. (17) Administration of methyldopa, which is the enzyme for synthesizing dopamine, may induce depression..$^{(3)}$ On the other hand there is evidence that administration of dopamine agonists may improve depression. ${ }^{(10)}$ Therefore, there is still a need for further studies in connection with the possibility of dopamine from blood samples being used as a biomarker of the level of depression or anxiety. The present study aimed to determine any correlation of blood dopamine level with the level of anxiety and depression in young adults.

\section{METHODS}

\section{Study design}

This study was of observational crosssectional design, using young adult subjects. The study was conducted from May to September 2018 in the Yogyakarta Special Region (Daerah Istimewa Yogyakarta).

\section{Study subjects}

The study subjects were adult males and females who agreed to participate in this study. The inclusion criteria were males or females, 18 up to 40 years of age, healthy, without severe illness. The exclusion criteria were lactating and pregnant females, smokers, consumption of LDopa or methyldopa. The condition of being healthy was determined from anamnesis that the respondent was in a condition of not having any symptom complaints or illness, and not having chronic disease.

\section{Determination of sample size}

The sample size for this study was calculated from the formula for sample size for correlation tests, which is $\{(Z \alpha+Z \beta) / 0.5 \ln [(1+r) /(1-r)]\}^{2}$ +3 , with an estimated moderate degree of correlation $(\mathrm{r}=0.6)$ and confidence level of $95 \%$, power of $80 \%$, with the addition of an anticipated number of drop-outs of $10 \%$. The result of the calculation showed that the minimal sample size was 32 . Recruitment of study subjects was by 
non-random consecutive sampling until the required minimal sample size was obtained. The investigators published the call for voluntary participation in the study through the Whatss App social media up to a given time limit and obtained 48 subjects. However, 5 subjects were prevented at the time of blood sample collection and ELISA assay, so that only data on 43 subjects were available for analysis.

\section{Determination of anxiety and depression scores}

The anxiety score is determined by means of the Beck Anxiety Inventory (BAI) questionnaire. The BAI score has been validated and its reliability tested, and may be used to determine the anxiety level in the clinic as well as in studies. ${ }^{(18)}$ The BAI questionnaire consists of 21 items, where each item uses a 4-point Likert scale (0-1-2-3). The scores range from 0 to 63 . Scores between $0-21$ indicate mild anxiety, scores 22-35 indicate moderate anxiety, while scores 36 and over indicate severe anxiety.

The depression scores are determined with the Beck Depression Inventory-II. This instrument has been proven to be of good validity and reliability and may be used to assess the depression level in populations of healthy individuals as well as in populations of individuals with disorders. ${ }^{(15,16)}$ The questionnaire consists of 21 items on a 4-point Likert scale of $0,1,2,3$ and total scores between 0 and 63 . Interpretation of the scores is as follows: scores 1-10 are normal scores, scores 11-16 indicate mild mood disorder, scores 17-20 mild depression, scores 21-30 moderate depression, scores 31-40 severe depression and scores higher than 40 extreme depression.

\section{Assessment of dopamine}

To each of the patients' venous blood samples EDTA was added, then the samples were centrifuged at $1000 \mathrm{G}$ for 20 minutes, and stored at $-80^{\circ} \mathrm{C}$ prior to the ELISA assay. The microplate wells were filled with an equal volume of serum sample and assayed according to the standard protocol of DA (dopamine) ELISA kit catalog No.U0392D028 (FineTest, Wuhan, Hupei, China). The results of the ELISA assay were read on an ELISA reader at a wavelength of 450 angstrom. Eight different concentrations of purified DA $(0,1.562 ; 3.125 ; 6.25 ; 12.5 ; 25,50$, and $100 \mathrm{ng} / \mathrm{ml}$ were used to construct the standard curve. The dopamine concentration was calculated with the formula (y-0.893)/ - 0.009.

\section{Statistical analysis}

Data on subject characteristics such as gender, education, and occupation were presented as total and frequency. Numerical data such as age, anxiety score, depression score, and dopamine score were presented as mean and standard deviation. The Kolmogorov-Smirnov test showed that the dopamine scores were normally distributed, but that the depression scores and anxiety scores were not. Therefore, the anxiety and depression scores were converted to an ordinal scale in accordance with the BAI guidelines, i.e. mild, moderate, and severe, and with the BDI guidelines, i.e. normal mood, mood disorder, and mild, moderate, severe, and extreme depression. Correlation was tested with the Spearman rho test. The level of significance used was 0.05 .

\section{Ethical clearance}

The present study obtained ethical clearance from the Ethics Committee, Faculty of Medicine, Universitas Islam Indonesia, under number 48/ Ka.Kom.Et/70/KE/V/2018. Prior to the start of the study, the study subjects signed written informed consent.

\section{RESULTS}

Among the subjects participating in this study there were more females $(62.8 \%)$ with mean age of 27 years. The most frequent educational level was S1 (48.8\%), and the most frequent occupation was employee (27.9\%) (Table 1). Although the dopamine concentrations were normally distributed, the results of the Kolmogorov- 
Table 1. Characteristics of study subjects $(n=43)$

\begin{tabular}{lcr}
\hline Characteristic & n (\%) & Mean ( \pm SD) \\
\hline Gender & $27(62.8)$ & \\
Female & $16(37.2)$ & \\
Male & & \\
Education & $15(34.9)$ & \\
Senior high school & $4(9.3)$ & \\
D3 & $21(48.8)$ & \\
S1 & $3(7)$ & \\
S2 & & \\
Occupation & $12(27.9)$ & $27.6 \pm 6.58$ \\
Employee & $10(23.2)$ & $15.44 \pm 12.04$ \\
Student & $5(11.6)$ & \\
Artist & $8(18.6)$ & \\
Unemployed & $8(18.6)$ & \\
Entrepreneur & & $12.56 \pm 33.32$ \\
Age (years ) & $34(79.0)$ & \\
Anxiety score & $6(14.0)$ & \\
Mild & $3(7.0)$ & \\
Moderate & & \\
Severe & $32(74.4)$ & \\
Depression scores & $7(16.3)$ & \\
Reasonable & $0(0.0)$ & \\
Mood disorder & $3(7.0)$ & \\
Mild depression & $1(2.3)$ & \\
Moderate depression & $0(0)$ & \\
Severe depression & & \\
Extreme depression & & \\
Dopamine concentration $(\mathrm{ng} / \mathrm{mL})$ & & \\
\hline
\end{tabular}

Smirnov test showed that the anxiety and depression scores were not normally distributed $(p>0.05)$. There was a tendency showing that the higher the anxiety and depression, the lower the dopamine concentrations. This was proven from the Spearman rho, which showed a negative correlation between blood dopamine level and degree of depression as well as between blood dopamine level and degree of anxiety, with a low coefficient of correlation for anxiety and a moderate correlation for depression. (Table 2).

\section{DISCUSSION}

The results of this study showed that there was a correlation between blood dopamine

Table 2. Correlation of dopamine level with degree of anxiety and depression

\begin{tabular}{lcc}
\hline & r* & p value \\
\hline Degree of anxiety & -0.310 & 0.043 \\
Degree of depression & -0.505 & 0.001 \\
\hline
\end{tabular}

*Spearman correlation : $r$ concentrations and severe degree of anxiety, although the correlation was weak. Dopamine is released when a person performs a pleasurable activity. On the other hand, a person with anxiety cannot feel pleasure. ${ }^{(20)}$ The results of the present study were in agreement with previous studies in experimental animals. Rat models of anxiety have lower dopamine levels than do normal rats. ${ }^{(21)}$ However, other studies obtained different results, since in patients with internet addiction disorder and concomitant anxiety, the blood dopamine levels were not significantly different from those of healthy controls. ${ }^{(17)}$ In contrast, studies in dogs found that plasma dopamine levels were higher in dogs with anxiety than in controls. ${ }^{(22)}$

Dopamine is involved in the regulation of pleasure, but it is also involved in the regulation of other factors such as addiction, motivation, and motor movement. There are four dopaminergic pathways in the brain, i.e. the nigro-striatal, mesolimbic, mesocortical, and tuberoinfundibular pathways. The mesolimbic pathway is involved 
in the regulation of pleasure. ${ }^{(23)}$ The blood dopamine level varies in the above-mentioned studies, because plasma dopamine level is also influenced by the dopamine secretion of sympathetic neurons, adrenal medulla, and neuroendocrine cells (amine precursor uptake and decarboxylation cells or APUD cells). These cells are found in the kidneys, leukocytes, and in both endocrine and exocrine glands of the pancreas. The blood dopamine level may increase due to stress, physical exercise, standing posture, and hypovolemia. This means that there are many factors that influence the blood dopamine level. Indeed, dopamine also plays an important role in glucose metabolism, weight regulation, and is said to play a role in protection against the proliferation of cancer. ${ }^{(24)}$

Although there is evidence that anxiety may increase in patients with Parkinson's disease after the cessation of dopaminergic medications, not all patients with Parkinson's disease experience improvement of anxiety after consumption of LDOPA (the precursor of dopamine). ${ }^{(25)}$ Increased dopamine in the synaptic cleft as a result of administration of dopamine reuptake inhibitors causes behavioral effects such as anxiety. ${ }^{(26)}$ In addition, other studies show that blood dopamine level cannot be equated with dopamine level in the brain. This is because dopamines in the blood cannot pass the blood brain barrier, ${ }^{(27)}$ except in the form of dopamine sulfate. The existing studies are more about central dopamines. Further studies need to be conducted in connection with peripheral dopamines.

The results of our study showed a moderate correlation between low dopamine level and severe depression. Prior studies on patients with post-stroke depression also showed lower dopamine levels as compared with those without depression (OR:0.64, 95\%CI: 0.45-0.91, $\mathrm{p}=0.014) .{ }^{(15)}$ However, other studies showed no correlation between BDI scores in 50 subjects with depression and blood dopamine level ${ }^{(28)}$ and no correlation of blood dopamine level with depression in patients with internet addiction disorder and concomitant depression in a study involving 20 patients and 15 controls. ${ }^{(17)}$ In contrast, in a study on 342 women postpartum, blood dopamine level in those with depression was higher than in those without depression. ${ }^{(16)}$ Similarly, studies on patients with major depressive disorder showed that the dopamine concentration as determined by gas chromatography-mass spectrometry (GC-MS) and liquid chromatography tandem mass spectrometry (LCMS/MS) was higher than in healthy controls. ${ }^{(6)}$ The existing studies on blood dopamine level and depression have still yielded variable results. In spite of this, there is evidence that administration of medications capable of increasing blood dopamine levels, such as tyrosine, amphetamine, and bupropion, may reduce the symptoms of depression. ${ }^{(23)}$ The regulation of dopamine secretion in the brain is rather complex. Dopamine is secreted by the pedunculopontine tegmental nucleus and the laterodorsal tegmentum by reward or appreciation stimuli. Activation of the ventral subiculum (vSub) of the hippocampus also induces increased dopamine secretion through the nucleus accumbens that inhibits the action of the ventral pallidum. However, if there is hyperactivity of the infralimbic subregion (ilPFC), this will activate the ventral pallidum via the basolateral amygdala, leading to a reduction in dopamine secretion, such as occurs in experimental animal models of depression. ${ }^{(13)}$

Dopamine neurotransmitters in the blood as biomarkers of depression are important for diagnosis and relatively simple to determine, but further studies are still necessary, because previous studies also showed that patients with medication-resistant depression who are treated with transcranial magnetic stimulation, experience increases in the level of dopamine metabolites in the brain, but there is no correlation between clinical improvement of depression and blood dopamine level. The measurement of neuroendocrine biomarkers such as dopamine is rather complex, in view of the rather widespread role of dopamine in the physiology of the body. ${ }^{(29)}$

The limitation of the present study is that it did not determine brain dopamine levels, that so 
far has been demonstrated to be more associated with the pathophysiology of anxiety and depression. Further studies need to be conducted in connection with peripheral dopamines and their connection with anxiety.

\section{CONCLUSION}

Low blood dopamine level in young adults is correlated with a higher degree of anxiety and depression, but the correlation is weak for anxiety and moderate for depression.

\section{ACKNOWLEDGMENTS}

The investigators thank the Ministry of Research and Technology Directorate of Higher Education (Kemenristek DIKTI) for the funding of this study in the framework of the Basic Research Grant Scheme For Higher Education (Skema Hibah Penelitian Dasar Perguruan Tinggi). The investigators also thank Ms Ernadita Budiatuti, technician in the Physiology Laboratory, Faculty of Medicine, Universitas Islam Indonesia, who assisted with blood sample collection and the ELISA assay.

\section{CONFLICT OF INTEREST}

There was no conflict of interest in this study.

\section{CONTRIBUTORS}

UK, AZA, TN, IF and ND all contributed equally to writing of the proposal, preparation and conduct of the study, analysis of the results, and writing and revising the manuscript. All authors have read and approved the final manuscript.

\section{REFERENCES}

1. World Health Organization. Depression and other common mental disorders: global health estimates. Geneva: World Health Organization; 2017.

2. Badan Penelitian dan Pengembangan Kesehatan Kementerian Kesehatan RI. Riset Kesehatan Dasar 2013. Jakarta: Badan Penelitian dan
Pengembangan Kesehatan Kementerian Kesehatan RI;2013.

3. Maina G, Mauri M, Rossi A. Anxiety and depression. J Psychopathol 2016;22:236-50.

4. Eskow JKL, George JA, Bishop C. L-DOPAinduced dysregulation of extrastriatal dopamine and serotonin and affective symptoms in a bilateral rat model of Parkinson's disease. Neuroscience 2012;;218:243-56.

5. Katie M. Smitha KM, Renshawb PF, et al. The diagnosis of depression: current and emerging methods. Compr Psychiatry 2013;54:1-6. doi: 10.1016/j.comppsych.2012.06.006.

6. Pan JX, Xia JJ, Deng FL, et al. Diagnosis of major depressive disorder based on changes in multiple plasma neurotransmitters: a targeted metabolomics study. Transl Psychiatry 2018;8:110. doi: 10.1038/s41398-018-0183-x

7. Lai CY, Yu SL, Hsieh MH, et al. MicroRNA expression aberration as potential peripheral blood biomarkers for schizophrenia. PLoS One 2011;6:e21635. doi: 10.1371/journal.pone.0021635.

8. Bocchio-Chiavetto L, Maffioletti E, Bettinsoli P, et al. Blood microRNA changes in depressed patients during antidepressant treatment. Eur Neuropsychopharmacol 2013;23:602-11. doi : 10.1016/j.euroneuro.2012.06.013.

9. Bonapersona V, Joëls M, Sarabdjitsingh RA. Effects of early life stress on biochemical indicators of the dopaminergic system: a 3 level meta-analysis of rodent studies. Neurosci Biobehav Rev 2018;95:1-16. doi: 10.1016/ j.neubiorev.2018.09.003.

10. Hori H, Kunugi H. Dopamine agonist-responsive depression. Psychogeriatrics 2013;13:189-95.

11. Bonomaully M, Khong T, Fotriadou M, et al. Anxiety and depression related to elevated dopamine in a patient with multiple mediastinal paragangliomas. Gen Hosp Psychiatry 2014;36: 449.e7-e8.

12. Robinson HM, Hood SD, Bell CJ, et al. Dopamine and social anxiety disorder. Rev Bras Psiquiatr 2006;28:263-4.

13. Belujon P, Grace AA. Dopamine system dysregulation in major depressive disorders. Int J Neuropsychopharmacol 2017;20:1036-46.

14. Tavakoli R, Nouri A, Masoudi R, et al. Serum markers related to depression: a systematic review. J Med Biomed Sci 2017;6:30-42. doi :10.4314/ jmbs.v6i3.5.

15. Meng G, Ma X, Li L, et al. Predictors of earlyonset post-ischemic stroke depression: a crosssectional study. BMC Neurol 2017;17:1-8.

16. Luo Y, Zheng L, Zhou J, et al. Relationship between the levels of estradiol and monoamine 
neurotransmitters and postpartum depression. Zhonghua Fu Chan Ke Za Zhi 2018;42:745-8.

17. Zhang HX, Jiang WQ, Lin ZG, et al. Comparison of psychological symptoms and serum levels of neurotransmitters in Shanghai adolescents with and without internet addiction disorder: a casecontrol study. PLoS One 2013;8:8-11.

18. Muntingh ADT, Van Der Feltz CM, Van Marwijk $\mathrm{HWJ}$, et al. Is the Beck anxiety inventory a good tool to assess the severity of anxiety? A primary care study in the Netherlands study of depression and anxiety (NESDA). BMC Fam Pract 2011;12:66.

19. Sorayah. Uji validitas konstruk Beck depression inventory II (BDI-II). J Pengukuran Psikol Pendidik Indones 2015;IV:29-44.

20. Zavos P. Women with anxiety and depression: out of the darkness and into the light. Women's Heal 2017;4:167-70.

21. Manikkoth SBD, Sequeira M, Elizabeth Joy A, et al. Assessment of brain dopamine levels to evaluate the role of Tylophora indica ethanolic extract on alcohol induced anxiety in Wistar albino rats. J Young Pharm 2016;8:91-5.

22. Riva J, Bondiolotti G, Michelazzi M, et al. Anxiety related behavioural disorders and neurotransmitters in dogs. Appl Anim Behav Sci 2008;114:168-81.

23. Ayano G. Dopamine: receptors, functions, synthesis, pathways, locations and mental disorders: review of literatures. J Ment Disord Treat 2016;2:2-5.

24. Rubí B, Maechler P. Minireview: new roles for peripheral dopamine on metabolic control and tumor growth: let's seek the balance. Endocrinology 2010;151:5570-81.

25. Picillo M, Santangelo G, Erro R, et al. Association between dopaminergic dysfunction and anxiety in de novo Parkinson's disease. Park Relat Disord 2017;37:106-10.

26. Nasehi M, Piri M, Nouri M, et al. Involvement of dopamine D1/D2 receptors on harmane-induced amnesia in the step-down passive avoidance test. Eur J Pharmacol 2010;634:77-83.

27. Suominen T, Piepponen TP, Kostiainen R. Permeation of dopamine sulfate through the blood-brain barrier. PLoS One 2015;10:e0133904.

28. Ambade V, Arora MM, Singh P, et al. Adrenaline, noradrenaline and dopamine level estimation in depression: does it help? Med J Armed Forces India 2009;65:216-20. doi: 10.1016/S03771237(09)80006-3.

29. Fidalgo T, Morales-Quezada L, Muzy G, et al. Biological markers in non-invasive brain stimulation trials in major depressive disorder: a systematic review. J Ect 2015;30:47-61. doi: 10.1097/YCT.0b013e31828b34d8. 